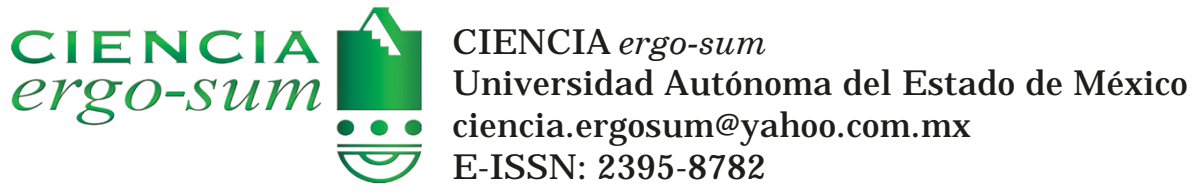

\title{
El internet en tiempos del SARS-Cov-2 (COVID-19) en México
}

\author{
García-Grajales, J .; Buenrostro-Silva, A.; López-Vázquez, Á. S. \\ El internet en tiempos del SARS-Cov-2 (COVID-19) en México \\ CIE NCIA ergo-sum, Número especial "SARS-CoV-2" 2021|e142 \\ Universidad Autónoma del Estado de México, México
}

Esta obra está bajo una Licencia Creative Commons Atribución-NoComercial-SinDerivar 4.0 Internacional .

García-Grajales, J ., Buenrostro-Silva, A. y López-Vázquez, Á. S. (2021). El internet en tiempos del SARS-Cov-2 (COVID-19) en México. https://doi .org/10.30878/ces.v28n4a3 


\title{
El internet en tiempos del SARS-Cov-2 (COVID-19) en México
}

\section{The Internet in times of SARS-Cov-2 (COVID-19) in Mexico}

\author{
Jesús Garcia-Grajales \\ Universidad del Mar, Campus Puerto Escondido, México \\ archosaurio@yahoo.com.mx \\ (D) http://orcid.org/0000-0001-6663-0388 \\ Alejandra Buenrostro-Silva \\ Universidad del Mar, Campus Puerto Escondido, México \\ sba1575@yahoo.com.mx \\ (D) http://orcid.org/0000-0003-3433-0668 \\ Ángel Salvador López-Vázquez \\ Universidad del Mar, Campus Puerto Escondido, México \\ angel.vazquez@outlook.com \\ (D) http://orcid.org/0000-0002-4473-0633
}

Recepción: 26 de enero de 2021

Aprobación: 18 de junio de 2021

\begin{abstract}
RESUMEN
Se presenta una reflexión sobre la problemática asociada con la calidad del internet en el país, sus consecuencias y retos en el proceso de educación a distancia mediada por la tecnología. Ante la situación de pandemia ocurrida durante 2020, el gobierno mexicano puso en funcionamiento varias medidas de mitigación, entre las que destaca el confinamiento voluntario en el hogar. Esta estrategia ha implicado un reto para la conectividad digital por el incremento exponencial del tráfico de internet. Por otro lado, todos los sistemas y niveles de educación han planteado la opción del uso de tecnologías de la información y las comunicaciones, en particular el internet de banda ancha, para continuar con el avance del aprendizaje.
\end{abstract}

PALABRAS ClaVE: banda ancha, conectividad digital, estrategias, satélite, pandemia.

\section{Abstract}

In this work we reflect on the problems associated with the quality of the Internet in Mexico, the consequence and challenges in the process of technology-mediated distance education. In view of the pandemic situation in 2020, the Mexican Government implemented several mitigation measures, including voluntary confinement. This strategy implies a challenge to digital connectivity due to exponential increase in Internet traffic. For other hand, all education systems have made the use of information and communication technologies as an option, in particular, the broadband Internet, to continue the advancement of learning.

KEYWORDS: broadband, digital connectivity, strategies, satellite, pandemic.

\section{INTRODUCCIÓN}

En diciembre de 2019 se identificó en Wuhan, China, una enfermedad (COVID-19) causada por un nuevo coronavirus denominado SARS-Cov-2 (Adnan et al., 2020; Lai, 2020; Palacios-Cruz et al., 2020; World Health Organization, 2020a). Posteriormente, el 7 de enero de 2020 la Organización Mundial de la Salud lo anunció de manera oficial como el agente causal de una serie de neumonías atípicas (World Health Organization, 2020a) que, en un porcentaje considerable, provocan la muerte de las personas infectadas (Huang et al., 2020).

Hasta el 29 de abril de 2020, ya se había extendido en 109 países, con un número de casos confirmados que superó los 3000000 y el registro de 207973 fallecimientos (World Health Organization, 2020b); esta situación 
hace que se catalogue como una enfermedad de emergencia en la salud pública mundial (Mahase, 2020) y, en consecuencia, en una pandemia (World Health Organization, 2020a).

En México el primer individuo contagiado se detectó el 20 de febrero de 2020 (Secretaría de Salud, 2020). Aproximadamente un año y tres meses después, el número de contagios confirmados se incrementó de manera alarmante a 2433681 personas y el de muertes a 228804 (World Health Organization, 2020b). Ante esta situación, el gobierno mexicano llevó a cabo varias acciones de intervención para mitigar la epidemia como fue la estrategia "Quédate en casa". Esta medida implicó el cierre de instituciones de educación, suspensión de labores no esenciales y la práctica de distanciamiento social incluida dentro de la Jornada Nacional de Sana Distancia, que además incluye la higiene profunda gracias al del lavado de manos y al uso del cubrebocas, así como el confinamiento voluntario promovido por el programa "Quédate en Casa" (Secretaría de Salud, 2020).

La pandemia de COVID-19 es inusitada, pues plantea un desafío al sistema socioeconómico mundial al afectar tanto a las economías avanzadas como a los países emergentes cuyas prácticas sociales y sistemas productivos se consideraban normales al final del 2019 (Agudelo et al., 2020). No obstante, las medidas sanitarias implementadas, principalmente las relacionadas con el confinamiento voluntario, implican un desafío a la infraestructura de telecomunicaciones y conectividad digital por el incremento exponencial del tráfico de internet, así como por el consecuente desafío para los operadores y empresas para mantener niveles de calidad óptimos por el incremento de la demanda (Sanz et al., 2020).

Por otro lado, todos los niveles del sistema de educación han planteado la opción del uso de tecnologías de la información y las comunicaciones (TIC), en particular el internet de banda ancha, para continuar con el avance del aprendizaje. Sin embargo, la calidad de los servicios de prestación de internet en México por región no es homogénea (Jordán et al., 2010), lo que genera complicaciones y retos para el cumplimiento de las actividades educativas a distancia mediadas por tecnología (Sanz et al., 2020).

Así, en este trabajo se presentan reflexiones sobre los problemas asociados con la calidad del internet en México, su costo y consecuencias en el proceso de educación a distancia. Además, se comenta cómo el rezago en materia de calidad del internet repercute en los procesos de enseñanza-aprendizaje a distancia durante la fase de mitigación de la pandemia del COVID-19.

\section{Las disparidades del internet en México}

Actualmente, es indiscutible que el internet ha revolucionado el papel de las telecomunicaciones y ha transformado la forma como se realizan las actividades cotidianas, por lo que se ha logrado eficientizar los procesos de generación e intercambio de información (Agudelo et al., 2020). Sin embargo, la imposición de medidas sanitarias para enfrentar el COVID-19 ha ocasionado un uso mayoritario de las redes de telecomunicación por parte de la población civil para resolver temas de aprovisionamiento de bienes, conectividad social y acceso a la información digital (Jordán et al., 2010; Katz et al., 2020). Este incremento en el uso de las redes de telecomunicaciones, como producto de las medidas de mitigación, ha generado una baja en la calidad de dichas redes, es decir, la velocidad promedio y latencia en internet disminuyeron de manera considerable en México durante las últimas semanas de marzo y el mes de abril (Ookla/Speedtest, 2020; Agudelo et al., 2020).

El desempeño del internet se mide en términos de a) la velocidad promedio de banda ancha fija (Mbps) y $b$ ) la latencia de banda ancha fija (ms). Sin entrar en un tecnicismo profundo, el ancho de banda se entiende como el acceso a internet con una capacidad igual o superior a $256 \mathrm{kbits} / \mathrm{s}$ en una o dos direcciones (subida y bajada) y existe en tres formas: $a$ ) banda ancha fija, $b$ ) banda ancha móvil y $c$ ) banda ancha inalámbrica; cada una cuenta con diferentes características tecnológicas, requerimientos de infraestructura y recursos humanos (Lazcano, 2017). Además, ésta se propaga a través de una red de telecomunicaciones, que es un sistema integrado por medios de transmisión tales como canales o circuitos que utilizan bandas de frecuencia del espectro radioeléctrico, enlaces satelitales, cableados, redes de transmisión eléctrica o cualquier otro medio de transmisión, así como centrales, 
dispositivos de conmutación o cualquier equipo necesario (Lazcano, 2017). Aunado a esto, la latencia se entiende como el tiempo que transcurre desde que un byte es enviado hasta que llega al otro extremo y viceversa, en tanto que la variación de la latencia (jitter) es un parámetro importante en la calidad, ya que afecta gravemente a aplicaciones multimedia tales como voz sobre IP. Las pérdidas o retardos son un indicador de la saturación de la red entre otros, ya que los routers y los equipos finales descartan paquetes cuando los reciben más rápido de lo que pueden procesar, lo cual implica una saturación (Miravalls, 2017).

Desde otro enfoque, la banda ancha se ha transformado en una infraestructura básica para el desarrollo económico y social, siendo el soporte de una amplia gama de actividades, desde el entretenimiento hasta el mundo laboral y la educación; por tanto, se está convirtiendo en una revolución digital al permitir la conectividad permanente y por ende en el desarrollo de oportunidades para la mejora de las comunicaciones orientadas a la prestación de servicios en línea (Jordán y De León, 2010).

Si bien el surgimiento de internet de banda ancha facilita aún más el proceso de intercambio de información permitiendo la conectividad permanente, así como el intercambio de un mayor flujo de contenidos y el acceso de calidad a servicios electrónicos avanzados que demandan la transferencia de grandes volúmenes de datos (audio, video, servicios interactivos, entre otros), la prestación de este servicio aún no logra superar las barreras económicas y geográficas que restringen la cobertura de servicios de interés social a los segmentos pobres y marginados (Jordán et al., 2010), y se generan entonces disparidades en la calidad del servicio de internet a lo largo del país (Miravalls, 2017).

En la administración del gobierno mexicano 2012-2018, la estrategia digital nacional denominada "Red compartida” fracasó en su intento de ampliar la cobertura de internet y telefonía en México, principalmente porque no se logró extender dicha cobertura más allá de las ciudades capital, tampoco logró acelerar la competencia en el sector de las telecomunicaciones ni influyó en la disminución de la brecha de conexión entre ricos y pobres pese a inversiones superiores a 140000 millones de pesos en el sector de las telecomunicaciones (Roldán, 2019; Arellano-Morales, 2020).

De acuerdo con la empresa privada Ookla/Speedtest ${ }^{[1]}$ que mide la velocidad promedio de banda ancha fija (Mbps) y la latencia de banda ancha fija ( $\mathrm{ms}$ ) con base en la compilación de pruebas realizadas por usuarios desde sus dispositivos, la velocidad y latencia del internet incrementó entre 50\% y 150\% (gráfica 1). Con el dato anterior se demuestra que la velocidad de banda ancha fija disminuyó gradualmente de finales de marzo hasta la última semana de abril de 2020 al compararlo con un periodo similar en el año anterior (2019), en tanto que la latencia de banda ancha fija incrementó de manera gradual en 2020, lo que representa una disminución en la calidad del servicio en el orden de 7.4\% para México (Agudelo et al., 2020). Todo esto se relaciona con el efecto de la estrategia "Quédate en casa", donde se promueven las actividades laborales en general y de enseñanza en modalidad de home office ("oficina desde casa") y con ello aumenta, en consecuencia, el tráfico en las redes de telecomunicaciones como producto del uso de las videoconferencias (Skype, Meet, Zoom, Facebook Live, YouTube Live, etc.), el mayor almacenamiento de información en la nube (Google Drive, Dropbox, OneDrive, etc.), la descarga de material digital para el aprendizaje (libros y artículos digitales), el mayor uso de las redes sociales (Facebook, Twitter, Instagram, WhatsApp) y de entretenimiento (Netflix, YouTube, Claro Video, etc.). Todos estos factores han creado en el último semestre de 2020 un "cuello de botella" de los enrutadores WiFi (Wireless Fidelity) que operan sobre el espectro no licenciado (generalmente en $2.4 \mathrm{GHz}$ y $5 \mathrm{GHz}$ ), con picos de tráfico en los horarios laborales y escolares. Lamentablemente, en las regiones donde la velocidad del internet es deficiente (menos de $1 \mathrm{Mbps)}$, como las áreas rurales de México, estas problemáticas se incrementan aún más. Por otro lado, las redes de telecomunicaciones dimensionan su capacidad con base en un uso promedio y no en picos de consumo; por tanto, el incremento al doble en la demanda de la red de telecomunicaciones en este periodo puede provocar problemas de saturación, generando la caída de los sistemas (Telmex, Megacable, etc.) y, en el peor de los casos, la avería de la red con su consecuente retraso en la reparación debido a la escasez de refacciones o componentes por el cierre de las fábricas (Agudelo et al., 2020). 


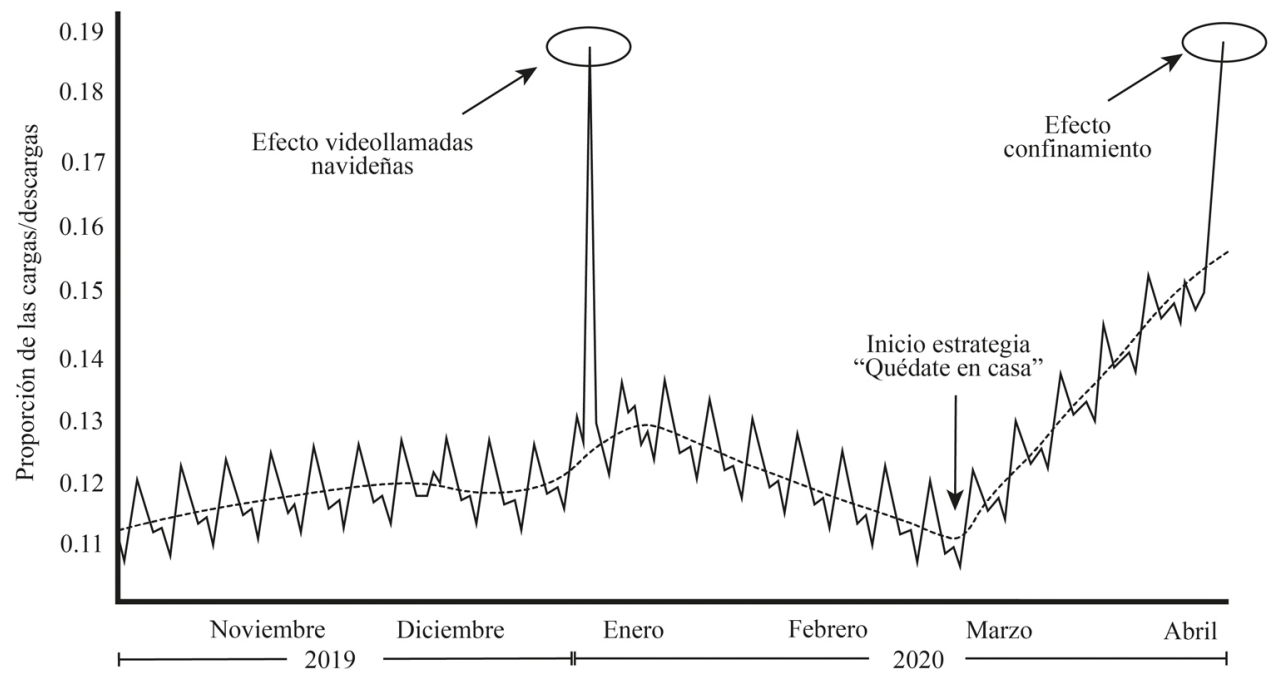

GRÁFICA 1

Demanda del consumo de internet en México en el periodo de noviembre de 2019 a abril de 2020 y el efecto del confinamiento

Fuente: elaboración a partir de las mediciones de Ookla/Speedtest, 2020.

En este sentido, la telefonía móvil mediante el uso del teléfono inteligente (smartphone) está desarrollando e incorporando, a una mayor velocidad, equipos multifuncionales (notebooks, smarphones, netbooks, tablet, PC, etc.), con capacidad de cómputo cada vez mayor, que permiten generar y transmitir grandes volúmenes de datos y videos sin olvidar su objetivo primordial, con pantallas más grandes, mejor calidad de resolución y teclados táctiles con formato QWERTY (teclado comúnmente utilizado y cuyo nombre proviene de las primeras letras de su fila de teclas) (Jordán et al., 2010).

Las nuevas generaciones comienzan a usar los smartphones con conexión a internet a edades muy tempranas (5 o 6 años). Por ello, la estrategia "Quédate en casa" y el cierre de los centros de educación (de todos los niveles) ha impulsado el uso de estos dispositivos con el consecuente aumento del tráfico de la banda ancha móvil, aún más cuando la banda ancha fija se satura o colapsa (Agudelo et al., 2020). De tal modo que la velocidad de banda ancha móvil y la latencia de banda ancha móvil presentan una tendencia de uso similar a los presentados por la banda ancha fija. No obstante, la banda ancha móvil se vuelve el elemento principal en aquellos sitios rurales donde no existe conectividad con la banda ancha fija; por lo tanto, la calidad del internet se vuelve fundamental porque determina también los servicios y aplicaciones a los que se puede acceder (Agudelo et al., 2020). Asimismo, el rezago de velocidad de la banda ancha móvil en relación con el promedio mundial de países más avanzados evidencia que los grupos y segmentos de la población mexicana que están excluidos de los beneficios de las tecnologías digitales (Lazcano, 2017), sobre todo en una situación como la actual pandemia, limitan las acciones efectivas del proceso enseñanza-aprendizaje, el trabajo desde casa y el esparcimiento.

\section{El internet EN TIEMPOS DEL COVID-19 Y LA BRECHA Digital}

La presencia del internet en los hogares es la palanca fundamental para poder afrontar la pandemia del COVID-19; ante esto, la digitalización del internet en los hogares permite a la población mexicana continuar realizando una cantidad de tareas cotidianas que con anterioridad requerían de contacto presencial (Agudelo et al., 2020). Sin embargo, en México aproximadamente $45 \%$ de la población desarrolla su principal actividad económica en el campo y por lo general viven en un entorno alejado de los centros urbanos, por lo que no tienen acceso a estos recursos y esto refleja la brecha digital existente. 
La brecha digital se define como la distancia tecnológica presente entre aquellos que tienen y no tienen acceso a las tecnologías, información e internet. Es una forma de exclusión entre los individuos, familias, empresas, comunidades y países que tienen acceso a las herramientas y capacidades para utilizar las tecnologías de la información y la comunicación (TIC) de aquellos que no las tienen (Lazcano, 2017). Esta brecha digital nacional muestra las diferencias entre los segmentos socioeconómicos, los niveles educativos o las comunidades dentro de un solo país; en consecuencia, se demuestran las desigualdades sociales, de recursos y oportunidades, la corrupción, entre otras (Lazcano, 2017).

De acuerdo con el INEGI, una comunidad rural se define cuando tiene menos de 2500 habitantes (Lazcano, 2017) y se caracteriza porque su población depende en mayor medida de la agricultura y la ganadería, así como pescadores, pastores nómadas o campesinos que se ocupan de la cría de animales, la transformación y comercialización de alimentos y otros productos y servicios derivados del agro (UNESCO, 2020). Adicionalmente, las comunidades alejadas se caracterizan por tener un desarrollo deficiente de los servicios básicos para sus habitantes como electricidad, educación, salud, agua potable y medios de comunicación (Lazcano, 2017). Además, sufren de aislamiento tecnológico que incrementa la brecha digital en México. Sin embargo, pese a las limitaciones mencionadas, han establecido modelos organizativos eficientes para sobrevivir al aislamiento económico, social, político y tecnológico, donde se han resguardado incluso sus rasgos culturales y lingüísticos, así como sus estructuras sociales (UNESCO, 2020).

Bajo este panorama de brecha digital en el país, la rapidez con la que se alteró la vida cotidiana de los humanos en el ámbito internacional, no sólo en México, planteó una situación inédita para todas las organizaciones, incluyendo las educativas a todos los niveles que se han visto obligados a suspender las actividades académicas presenciales en una transición abrupta y obligada a la educación a distancia mediada por tecnología (Sanz, 2020).

La suspensión obligatoria de clases en todos los niveles educativos, con el confinamiento en casa de estudiantes y profesores, ha creado una red de efectos múltiples (como el acondicionamiento de espacios en casa para la impartición o toma de clases) en todos los actores del proceso educativo, y se han tomado como medidas paliativas el uso de la infraestructura informática y tecnológica que les permita continuar con sus actividades académicas a distancia (Sánchez Mendiola et al., 2020). Sin embargo, el confinamiento, la presencia o carencia de dispositivos digitales, las presiones propias de la convivencia en familia, así como la velocidad de internet del que dispongan tanto profesores como estudiantes, presuponen una compleja situación simultánea de acciones y emociones que no se habían experimentado con anterioridad (Sánchez Mendiola et al., 2020; Sanz, 2020).

De este modo, es como la brecha digital enfatiza su presencia debido a que la migración hacia el proceso de enseñanza aprendizaje a distancia no servirá para aquellas comunidades rurales con carencia o mala calidad del internet, por lo que las tareas, conferencias virtuales, transferencias de información, entre otras, sufrirán un rezago y en la mayoría de los casos una ausencia de los profesores y alumnos (Lazcano, 2017).

\section{Consecuencias y Retos ANTE LA BRECHa digital DEL INTERNET EN TIEMPOS DEL COVID-19}

El acceso universal al internet es la posibilidad de que todos los miembros de una población tengan entrada a los servicios de la red de comunicación de carácter público, comunitario o compartido (Lazcano, 2017). En este sentido, Oestmann y Dymond (2009) mencionan que existen tres elementos fundamentales para alcanzarla: $a$ ) disponibilidad, es decir, que los servicios deben encontrarse en las zonas poco pobladas de un país a través de dispositivos públicos, comunitarios, compartidos o personales, $b$ ) accesibilidad, entendida como el hecho de que todos los ciudadanos puedan utilizar el servicio sin importar su ubicación geográfica ni su nivel socioeconómico, discapacidades u otras características personales y $c$ ) asequibilidad, referente a que el servicio debe ser alcanzable para todos los ciudadanos. 
Ante esta panorma, la rapidez con la que se expandió la pandemia del COVID-19 ha generado una repentina nueva realidad y una situación inesperada en la educación de todos los niveles; sin embargo, el principal reto ahora es encontrar estrategias de enseñanza y aprendizaje que logren vencer a la brecha digital existente en nuestro país. No obstante, el reto de la educación a distancia varía sustancialmente por nivel educativo, es decir, las modalidades de enseñanza cambian para bachillerato, licenciatura o posgrado, ya que la autonomía para el aprendizaje depende de las experiencias previas en la escolaridad, el nivel de conocimientos y el dominio de habilidades, así como la estructura y el nivel de profundidad de los planes y programas de estudio, el método de enseñanza y el número de alumnos por grupo (Sánchez Mendiola et al., 2020). Aunado a esto, las problemáticas logísticas y tecnológicas vinculadas a la muralla virtual de la pobre infraestructura de las comunicaciones en México, así como la brecha digital existente entre los diversos sectores de la sociedad (tanto en docentes como en estudiantes), hacen prácticamente imposible realizar educación mediada por tecnología.

\section{Prospectiva}

El cambio de la clase tradicional presencial en el salón que por décadas ha practicado la mayoría de los profesores, los ha convertido, de manera inusitada y obligada, en usuarios de herramientas tecnológicas a distancia. Lo anterior nos hace reflexionar sobre la necesidad de participar en actividades de formación docente que impliquen adiestramiento en el uso de tecnologías para el beneficio profesional, personal y de los estudiantes (Sánchez Mendiola et al., 2020). Además, la brecha digital existente en el país impide que un grupo significativo de alumnos tenga acceso a las clases a distancia, por lo que, en este caso, la empatía del profesorado será una capacidad valorada en estos tiempos.

\section{REFERENCIAS}

Adnan, M., Khan, S., Kazmi, A., Bashir, N., \& Siddique, R. (2020). COVID-19 infection: Origin, transmission, and characteristics of human coronaviruses. Journal of Advanced Research, 24, 91-98. https://doi. org/10.1016/j.jare.2020.03.005

Agudelo, M., Chomali, E. y Suniaga, J. (2020). Las oportunidades de la digitalización en América latina frente al COVID-19. Corporación Andina de Fomento/Naciones Unidas.

Arellano-Morales, M. A. (2020). Las brechas digitales en México: un balance pertinente. El Trimestre Económico, 346(2), 367-402.

Huang, C., Wang, Y., Li, X., Ren, L., Zhao, J., ... Hu, Y.(2020). Clinical features of patients infected with 2019 novel coronavirus in Wuhan China. The Lancet, 395, 497-506. https://doi.org/10.1016/S0140-6736(20)30183.5

Jordán, V., Galperin, H. y Peres, W. (2010). Acelerando la revolución digital: banda ancha para América latina y el Caribe. Santiago de Chile: Naciones Unidas.

Jordán, V. y De León, O. (2010). La banda ancha y la concreción de la revolución digital, en V. Jordán, V., H. Galperin y W. Peres (eds.), Acelerando la revolución digital: banda ancha para América latina y el Caribe (pp. 13-50). Santiago de Chile: Naciones Unidas.

Katz, R. L. (2010). La contribución de la banda ancha al desarrollo económico. In Jordán, V., Galperin, H., Peres, W. (eds.) Acelerando la revolución digital: banda ancha para América latina y el Caribe (pp. 51-84). Santiago de Chile: Naciones Unidas.

Katz, R., L., Jung, J. y Callorda, F. (2020). Estado de la digitalización en América latina frente a la pandemia. Observatorio del Ecosistema digital de América Latina y el Caribe. CAF. 
Lai, C. C., Ko, W. C., Tang, H. J., \& Hsueh, P. R. (2020). Severe acute respiratory syndrome coronavirus 2 (SARS-Cov-2) and coronavirus disease-2019 (COVID-19): The epidemic and the challenges. International Journal of Antimicrobial Agents, 55(3), 1-9. https://doi.org/10.1016/j.ijantimicag.2020. 1059924.

Lazcano, R. (2017). Inclusión de comunidades geográficamente alejadas en México a través de las telecomunicaciones digitales y servicios de banda ancha (tesis de licenciatura). México: Universidad Nacional Autónoma de México.

Mahase, E. (2020). China coronavirus: WHO declares international emergency as death toll exceeds 200. BMJ, 368, m408. https://doi.org/10.1136/bmj.m408

Miravalls, E. (2017). Análisis de métricas ligeras indicadoras de la calidad en internet (tesis de maestría). Madrid: Escuela Politécnica Superior.

Oestmann, S. y Dymond, A. (2009). Acceso y servicio universal (ASU). Disponible en: http:/ictrregulationtoolkit. org/Documents/Document/3733

Ookla/Speedtest (2020). Tracking COVID-19's impact on Global Internet Performance. Retrieved from https:// www.speedtest.net/insights/blog/tracking-covid-19-impact-global-internet-performance/\#/Mexico

Palacios-Cruz, M., Santos, E., Velázquez, M. A. y León, M. (2020). COVID-19, una emergencia de salud pública mundial. Revista Clinica Española. https://doi.org/10.1016/j.rce.2020.03.001

Roldán, N. (2019). El proyecto de Peña Nieto para ampliar la cobertura de internet no generó competencia ni redujo tarifas. Animal Politico. Disponible en: https://www.animalpolitico.com/2019/11/pena-nieto-internet-red-compartida-competencia-raifas/

Sánchez Mendiola, M., Martínez, A. M. P., Torres, R., Agüero, M., Hernández, A., Benavides, M. A., Jaimes, C. y Rendón, V. (2020). Retos educativos durante la pandemia de COVID-19: una encuesta a profesores de la UNAM. Revista Digital Universitaria, 1-23.

Sanz, I., Sainz, J. y Capilla, A. (2020). Efectos de la crisis del coronavirus en la educación superior. Organización de Estados Iberoamericanos para la Educación, la Ciencia y la Cultura. Disponible en: https://oei.org.br/ arquivos/informe-covid-19d.pdf

Secretaría de Salud. (2020). Comunicado técnico diario COVID-19 MÉXICO. Subsecretaría de Prevención de la Salud. Disponible en: https://www.gob.mx/cms/uploads/attachment/file/549621/Comunicado_Tecnico_Diario_COVID-19_2020.04.29.pdf

UNESCO. (2020). ICTs and indigenous people. Retrieved from http://iite.unesco.org/pics/publications/en/ files/3214689.pdf

World Health Organization. (2020a). Novel Coronavirus (2019-nCoV). Situation Report-1. Consultado el 28 de abril de 2020. Disponible en https://www.who.int/docs/default-source/coronaviruse/situationreports/20200121-sitrep-1-2019-ncov.pdf ?sfvrsn=20a99c10_4

World Health Organization. (2020b). Coronavirus disease 2019 (COVID-19). Situation report-100. Consultado el 30 de abril de 2020. Disponible en: https://www.who.int/docs/default-source/coronaviruse/ situation-reports/20200429-sitrep-100-covid-19.pdf ?sfvrsn=bbfbf3d1_2

Nota

[1] Disponible en https://www.speedtest.net

CC BY-NC-ND 